\title{
Endoscopic ultrasound (EUS)-guided fine needle biopsy alone vs. EUS-guided fine needle aspiration with rapid onsite evaluation in pancreatic lesions: a multicenter randomized trial
}

Authors

Yen-I Chen ${ }^{1} \odot$, Avijit Chatterjee ${ }^{2}$, Robert Berger ${ }^{3}$, Yonca Kanber ${ }^{4}$, Jonathan Wyse ${ }^{5}$, Eric Lam ${ }^{6}$, Ian Gan ${ }^{7}$, Manon Auger ${ }^{4}$, Sana Kenshil ${ }^{2}$, Jennifer Telford ${ }^{6}$, Fergal Donnellan ${ }^{7}$, James Quinlan², Gregory Lutzak ${ }^{8}$, Fatma Alshamsi², Josee Parent ${ }^{1}$, Kevin Waschke ${ }^{1}$, Adel Alghamdi ${ }^{1}$, Jeffrey Barkun ${ }^{9}$, Peter Metrakos ${ }^{9}$, Prosanto Chaudhury ${ }^{9}$, Myriam Martel ${ }^{1}$, Alastair

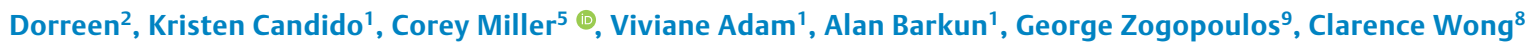

Institutions

1 Division of Gastroenterology and Hepatology, McGill University Health Centre, Montreal, Quebec, Canada

2 Division of Gastroenterology and Hepatology, Ottawa Hospital, Ottawa University, Ottawa, Ontario, Canada

3 Division of Gastroenterology, Moncton Hospital, Moncton, New Brunswick, Canada

4 Department of Pathology, McGill University Health Centre, Montreal, Quebec, Canada

5 Division of Gastroenterology and Hepatology, Jewish General Hospital, McGill University, Montreal, Quebec, Canada

6 Division of Gastroenterology and Hepatology, St-Paul Hospital, Vancouver, British Columbia, Canada

7 Division of Gastroenterology and Hepatology, Vancouver General Hospital, Vancouver, British Columbia, Canada

8 Division of Gastroenterology and Hepatology, Royal Alexandra Hospital, University of Alberta, Edmonton, Alberta, Canada

9 Department of Surgery, McGill University Health Centre, Montreal, Quebec, Canada

submitted 10.9.2020

accepted after revision 27.1.2021

published online 27.1.2021

Bibliography

Endoscopy 2022; 54: 4-12

DOI 10.1055/a-1375-9775

ISSN 0013-726X

(c) 2021. Thieme. All rights reserved.

Georg Thieme Verlag KG, Rüdigerstraße 14,

70469 Stuttgart, Germany

Corresponding author

Yen-I Chen, MD, Division of Gastroenterology and

Hepatology, McGill University Health Centre, Montreal, QC,

Canada

yen-i.chen@mcgill.ca

\section{ABSTRACT}

Background Endoscopic ultrasound-guided fine-needle aspiration (EUS-FNA) is the standard in the diagnosis of solid pancreatic lesions, in particular when combined with rapid onsite evaluation of cytopathology (ROSE). More recently, a fork-tip needle for core biopsy (FNB) has been shown to be associated with excellent diagnostic yield. EUS-FNB alone has however not been compared with EUS$\mathrm{FNA}+\mathrm{ROSE}$ in a large clinical trial. Our aim was to compare EUS-FNB alone to EUS-FNA+ROSE in solid pancreatic lesions.

Methods A multicenter, non-inferiority, randomized controlled trial involving seven centers was performed. Solid pancreatic lesions referred for EUS were considered for inclusion. The primary end point was diagnostic accuracy. Secondary end points included sensitivity/specificity, mean number of needle passes, and cost.

Results 235 patients were randomized: 115 EUS-FNB alone and 120 EUS-FNA+ROSE. Overall, 217 patients had malignant histology. The diagnostic accuracy for malignancy of EUS-FNB alone was non-inferior to EUS-FNA + ROSE at $92.2 \%(95 \% \mathrm{Cl} 86.6 \%-96.9 \%)$ and $93.3 \%(95 \% \mathrm{Cl} 88.8 \%-$ $97.9 \%)$, respectively $(P=0.72)$. Diagnostic sensitivity for malignancy was $92.5 \%(95 \% \mathrm{Cl} 85.7 \%-96.7 \%)$ for EUS-FNB alone vs. $96.5 \%(93.0 \%-98.6 \%)$ for EUS-FNA+ROSE ( $P=$ 0.46 ), while specificity was $100 \%$ in both. Adequate histological yield was obtained in $87.5 \%$ of the EUS-FNB samples. The mean (SD) number of needle passes and procedure time favored EUS-FNB alone (2.3 [0.6] passes vs. 3.0 [1.1] passes $[P<0.001]$; and 19.3 [8.0] vs. 22.7 [10.8] minutes $[P=0.008])$. EUS-FNB alone cost on average 45 US dollars more than EUS-FNA + ROSE.

Conclusion EUS-FNB alone is non-inferior to EUS-FNA+ ROSE and is associated with fewer needle passes, shorter procedure time, and excellent histological yield at comparable cost. 


\section{Introduction}

Endoscopic ultrasound (EUS)-guided tissue acquisition is the standard of care for the diagnosis of solid pancreatic lesions $[1,2]$. Traditionally, sampling is performed through fine-needle aspiration (FNA) and the diagnosis is made through cytology. The challenge in solid pancreatic lesions is that they are often fibrotic, with significant necrosis limiting the cellularity of samples obtained via FNA. As such, some tertiary institutions have adopted rapid onsite evaluation of cytopathology (ROSE) to attain a diagnostic yield of $>90 \%[3,4]$. ROSE however requires considerable expertise and its availability is limited outside of the most expert centers.

Although efforts have been made in the past two decades to develop core biopsy needles or cutting needles for histological assessment, it was not until recently, with the advent in 2016 of the fork-tip needle (SharkCore, Medtronic, USA) and in 2017 of the Franseen tip needle (Acquire, Boston Scientific, USA), that truly reliable fine-needle biopsy (FNB) devices emerged. Randomized controlled trials (RCTs) have now demonstrated a diagnostic yield of greater than $90 \%$ with both FNB needles and excellent histological yield $[5,6]$, which may play an increasingly important role for ancillary molecular analysis and personalized medicine. The important question however of whether FNB requires the presence of ROSE remains to be elucidated and it is unclear whether EUS-FNB alone is comparable to EUSFNA with ROSE.

In this multicenter RCT, we aimed to ascertain the diagnostic performance of EUS-FNB alone vs. EUS-FNA + ROSE in solid pancreatic lesions. To our knowledge, although trials have emerged comparing FNB with FNA, this is the first reported multicenter RCT to directly assess the need for ROSE in the era of biopsy needles for solid pancreatic lesions.

\section{Methods}

This was a multicenter, randomized, non-inferiority trial comparing EUS-FNB alone to EUS-FNA + ROSE in the diagnosis of solid pancreatic masses. We hypothesized that EUS-FNB alone is non-inferior to EUS-FNA + ROSE in terms of diagnostic accuracy for solid pancreatic lesions, while being associated with greater histological yield, fewer needle passes, and shorter procedure time, with comparable cost. Overall, seven Canadian centers ( 6 tertiary and 1 secondary center) across five provinces participated in the study. This was a registered study approved by the research ethics board at the coordinating site and all participating centers. All authors had access to the study data, and reviewed and approved the final manuscript.

\section{Study population}

All patients referred for EUS assessment and tissue diagnosis of a solid pancreatic lesion were considered for enrollment. The inclusion criteria were age $>18$ years and referral for EUS evaluation of a definite solid pancreatic mass noted on computed tomography (CT) or magnetic resonance imaging (MRI) or EUS, in which malignancy was suspected, with no previous histological diagnosis. The exclusion criteria were: pregnancy; uncorrect- able coagulopathy (PTT $>50$ seconds or INR $>1.5$ ) and/or uncorrectable thrombocytopenia (platelet count $<50 \times 109 /$ L); lesions that were deemed inaccessible for EUS-guided sampling; patients who were too clinically ill to undergo an EUS examination.

\section{Randomization}

After they had given consent, patients were randomized to undergo either EUS-FNB alone or EUS-FNA + ROSE. Randomization was performed during the procedure once the endoscopist had confirmed the presence of a solid pancreatic lesion that was safely accessible via EUS. To ensure allocation concealment, an online randomization software (randomize.net) was used by the local research staff. Included subjects were randomized in a 1:1 ratio in blocks of 4 stratified by endoscopist experience (assessed as career linear EUS procedures of 500-1000, 10002000, 2000-3000, and >3000).

Both the research personnel involved in the statistical analysis and the patients, but not the endoscopists, were blinded. Pathologists were not blinded to the allocation as this was not feasible, given the difference in tissue processing between FNA and FNB samples.

\section{Treatment protocol}

All procedures were undertaken by an experienced endoscopist who had performed at least 500 linear EUS procedures. After informed consent had been obtained, all EUS examinations were performed with the patient under endoscopist-directed conscious sedation. The use of antibiotics was at the discretion of the endoscopist.

\section{EUS-FNB alone}

A curvilinear echoendoscope was inserted orally and advanced to the stomach or duodenum. Once the solid pancreatic lesion was located, color Doppler was applied to ensure the safety of needle puncture. Tissue acquisition was performed with a 22or 25-gauge fork-tip FNB needle, in a transgastric or transduodenal fashion, using the fanning technique. The use of adjuvant tissue acquisition methods including suction, wet suction, and slow pull were at the discretion of the endoscopist. Two passes were performed with the samples expressed using a stylet into a jar filled with $10 \%$ formalin. A third pass was allowed if the macroscopic onsite evaluation of the biopsy specimen (MOSE) was deemed insufficient by the endoscopist. For MOSE, the endoscopist inspected the acquired sample inside the formalin jar following the first two passes and deemed the specimen to be sufficient if pink core tissue was noted. If no clear tissue was visualized and/or the sample was deemed to be only blood, a third and final needle pass was then performed.

\section{EUS-FNA with ROSE}

EUS-FNA + ROSE was performed with 22- or 25-gauge FNA needles using the same technique described above. The sampled specimen was expressed into a glass slide with a stylet and the sample was spread out using another glass slide to make smears on two slides. Each pair of slides was then numbered according to their respective needle passes. One slide was air-dried and 
stained with the Diff-Quik stain for ROSE, while the other slide was fixed with cytospray to be stained later with the Papanicolaou stain in the cytopathology laboratory. Any additional tissue from each pass was expressed at the time of the ROSE procedure into a jar filled with $10 \%$ Cytolyt. After completion of the procedure and upon return to the cytopathology laboratory, an additional cytospin slide was prepared from this amalgamated material and only the rest of the sample was used for a cell block.

Owing to a lack of robust data supporting one needle size or one sampling technique over another $[2,7]$, the needles size and tissue acquisition technique were left to the discretion of the endoscopist. This approach was intended to mimic realworld practice, wherein variations in needle sizes and sampling techniques exist [8].

\section{Study end points}

The primary end point was diagnostic accuracy, defined as (true positive + true negative)/all samples.

A final diagnosis of malignancy for pancreatic exocrine tumors was defined and modified from previous published criteria [9] as FNA or FNB cytopathology samples reported as either suspicious for malignancy or definite for malignancy, and one of the following: (1) histological evidence of malignancy on the corresponding subsequent surgical specimen, (2) presence of an observed unresectable lesion during subsequent surgery, (3) locoregional progression/development of metastases on follow-up axial imaging, (4) stability or improvement of the lesion on axial imaging with chemotherapy, and/or (5) cancerrelated death or hospice care within 6 months of identification of the mass. For non-exocrine pancreatic cancers, such as neuroendocrine tumor and lymphoma, FNA or FNB cytopathological diagnosis was deemed diagnostic for malignancy on its own. The definitions of malignancy for non-exocrine pancreatic cancer were added following trial commencement as they did not fit the criteria for exocrine tumor.

The definition for a final diagnosis of benign disease was: FNA or FNB samples reported as atypical or negative for malignancy, and one of the following: (1) surgical pathology or exploration showing absence of malignancy, (2) follow-up imaging after $>6$ months reporting stability or improvement of the pancreatic lesion without receiving chemotherapy, (3) cytological or histopathological diagnosis of benign disease with an appropriate clinical course of disease for a minimum of 6 months.

Secondary outcomes included diagnostic characteristics (sensitivity, specificity, and positive and negative predictive values); mean number of needle passes; and procedure time, defined as the time from endoscope insertion to endoscope withdrawal.

Additional outcomes included: adverse events as per the ASGE lexicon [10]; cost-minimization data; and specimen adequacy for histological assessment (histology score $\geq 3$ ). Adequacy of histological interpretation was based on a standardized score [11]: 0, insufficient material for interpretation; 1 , sufficient material for limited cytological interpretation; 2 , sufficient material for adequate cytological interpretation; 3 , suffi- cient material for limited histological interpretation; 4, sufficient material for adequate histological interpretation, low quality (total material length of less than a $10 \times$ power field); and 5 , sufficient material for adequate histological interpretation, high quality (longer than a $10 \times$ power field).

\section{Data collection and follow-up}

The data collected included: the primary and secondary end points; demographics; laboratory investigations, including liver enzymes, complete blood count, coagulation profile, and CA19-9; tumor location and staging on axial imaging (CT or $M R I)$; findings on EUS, including the presence of a biliary stent, tumor size, margins, and echogenicity; size of needle used; sampling technique, including slow pull, and dry or wet suction; tumor consistency; number of needle passes; procedure time, defined as the time from endoscope insertion to endoscope withdrawal.

Patients were contacted by telephone and interviewed within 7 days post-procedure to capture any adverse events. Follow-ups were then scheduled at days 30, 90, and 180.Patients were followed until the primary end point had been reached, death occurred, or a total of 6 months follow-up had been completed.

All authors reviewed and approved the final manuscript and had access to the study data.

\section{Sample size}

The primary analysis was a non-inferiority comparison of EUSFNB alone vs. EUS-FNA + ROSE in terms of diagnostic accuracy, which, according to the most recent literature, is approximately $90 \%$ for both modalities. The margin of non-inferiority was set at $10 \%$ in accordance with the US FDA recommendations. To achieve a statistical power of $80 \%$, with a one-sided type I error of $5 \%$, based on the normal approximation test of proportions, a total of 224 patients (112 per group) was needed (nQuery). Assuming a $5 \%$ dropout rate, a final sample size of 236 (118 per group) was estimated to be required.

\section{Statistical analysis}

Descriptive data were generated; categorical data were expressed as proportions, and continuous data were expressed as mean (standard deviation [SD]). The chi-squared test or Fisher exact test for categorical variables and t test or non-parametric Wilcoxon signed-rank test for continuous variables were used where appropriate to compare baseline demographics and the FNA vs. FNB modalities. An intention-to-treat analysis was carried out for diagnostic accuracy, sensitivity, and specificity; the non-inferiority hypothesis for this primary outcome was assessed using the $Z$ test, with a $95 \%$ one-sided confidence interval $(\mathrm{Cl})$. A standard stepwise multivariable logistic regression analysis was performed to identify predefined variables that were associated with diagnostic accuracy; results were presented as odds ratios (ORs) with $95 \% \mathrm{Cls}$. A two-sided P value of $<0.05$ was considered statistically significant. Statistical analyses were performed with SAS (version 9.4, 2016; SAS Institute Inc., Cary, North Carolina, USA). 


\section{Cost analysis}

Based on the data from our RCT, a cost-minimization analysis was performed comparing EUS-FNB alone with EUS-FNA plus ROSE in the USA and Canada [12]. The average cost per intervention was the result of the sum of the technical (specific medical material) and professional (physician, nurse, and cytotechnician) components. The cost of the microscope for ROSE (Olympus BX46TF; Olympus Medical, Japan) was amortized to obtain an equivalent cost per single use. We assumed that EUS-FNB requires the endoscopist and one nurse, while EUSFNA with ROSE requires the endoscopist, one nurse, and one cytotechnician. In the US setting, we also assumed the presence of an anesthesia nurse and anesthesia physician. No anesthesia fees were included in the Canadian setting, given that nearly all EUS procedures are performed with the patient under endoscopist-guided conscious sedation. We did not include professional fees for a cytopathologist or any costs associated with the processing of the samples in the pathology laboratory. Institutional fees were not included, given that they are likely to be the same for both arms.

All costs were expressed in 2019 US dollars (USA) and 2019 CAN dollars (Canada) and presented as the marginal cost difference between the two interventions. Deterministic sensitivity analyses were performed varying all associated costs leading to the minimum and the maximum differences between the two modalities.

US physician fees (gastroenterologist and anesthetist) were based on national US data from the Centers for Medicare and Medicaid Services [13]. National yearly hourly wages for cytotechnicians and nurses were provided by the US Bureau of Labor Statistics [14]. National market prices of the needles and microscope were obtained from Medtronic Inc. and Olympus Inc., respectively. Canadian gastroenterologist fees were based on data from the Schedule of Benefits of Ontario Ministry of Health [15]. National hourly wages for cytotechnicians and nurses were provided by Statistics Canada [16]. Needle and microscope prices were obtained from industry and the McGill University Health Centre purchasing office [17]. Mean and SD of the procedure times were obtained from the RCT.

\section{Results}

Overall, 235 patients were successfully randomized with 115 patients in the FNB arm and 120 in the FNA arm ( $\triangleright$ Fig. 1). Recruitment for the trial was performed from February 2018 to January 2020, with completion of follow-up in June 2020. The primary end point of diagnostic accuracy was reached in 114 EUS-FNB patients (one patient lost to follow-up) and 118 EUSFNA + ROSE patients (two lost to follow-up).

The mean (SD) age of the patients was 69.7 (11.8) years with $47.7 \%$ of the patients being women ( $\triangleright$ Table 1 ). Adenocarcinoma was the most common tumor histology (79.7\%), followed by pancreatic neuroendocrine tumor (7.8\%), metastatic cancer to the pancreas $(4.6 \%)$, lymphoma (1.4\%), solid pseudopapillary neoplasm (1.4\%), and other (1.8\%). Only $18.9 \%$ of patients underwent surgical resection, while $59.1 \%$ of patients received
Assessed for eligibility ( $n=236$ )

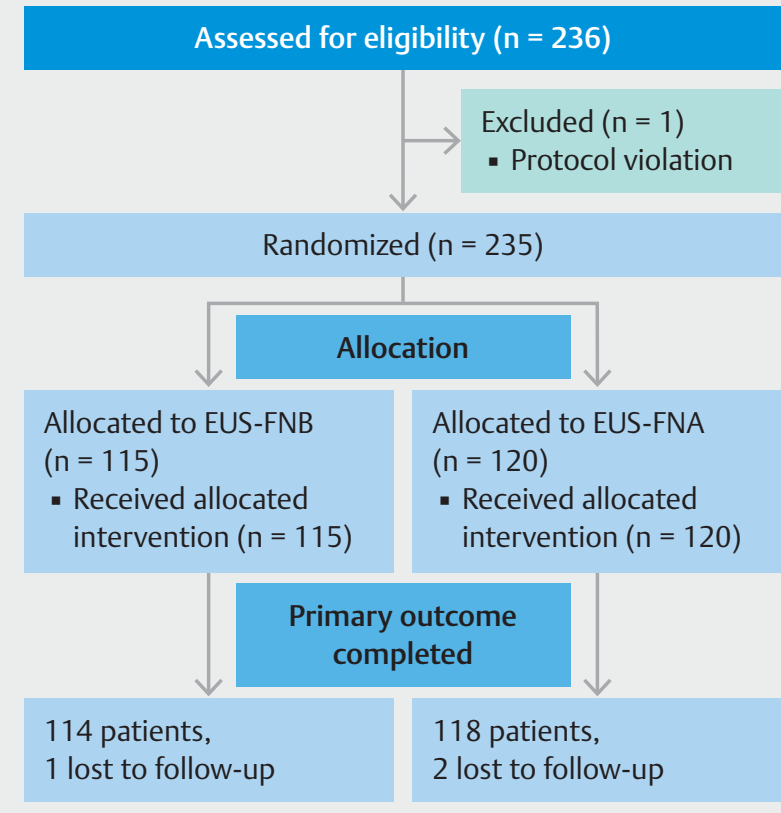

- Fig. 1 Consort diagram showing the flow of patients though the study.

chemotherapy treatment, with $27.0 \%$ of patients dying during the 6 months follow-up. Except for a slightly more predominant rate of Stage IV cancer in the EUS-FNA + ROSE cohort, no other significant differences in baseline characteristics were noted.

On EUS examination, the mean (SD) tumor sizes were 30.4 (13.3) $\mathrm{mm}$ and $29.3(12.0) \mathrm{mm}$ for EUS-FNB alone and EUSFNA + ROSE, respectively $(P=0.52)$ ( $($ Table 2$)$. In terms of needle size, $95.6 \%$ of EUS-FNBs were performed with a 22-gauge FNB needle and $4.2 \%$ with a 25 -gauge FNB needle. In the EUSFNA+ROSE arm, $44.0 \%$ used a 22-gauge needle and $56.0 \%$ used a 25-gauge needle. EUS-FNB required fewer needle passes (mean [SD], 2.3 [0.6] vs. 3.0 [1.1]; $\mathrm{P}<0.001$ ) The mean (SD) procedure time was also significantly shorter with EUS-FNB at 19.3 (8.0) minutes vs. 22.7 (10.8) minutes $(P=0.008)$. The overall adverse event rate was low at $1.3 \%$ (0.0\% EUS-FNB, 2.5 $\%$ EUS-FNA ROSE). These adverse events were rated as moderate (one pancreatitis) and severe (one pancreatitis and one bleed).

The primary outcome of diagnostic accuracy was $92.2 \%$ for EUS-FNB alone and $93.3 \%$ for EUS-FNA + ROSE $(P=0.72)(>$ Table 3). Diagnostic accuracy did not vary significantly with endoscopist experience. Diagnostic sensitivity was also comparable between the two modalities at $92.5 \%$ with EUS-FNB alone vs. $96.5 \%$ with EUS-FNA + ROSE $(P=0.46)$. The specificity and positive predictive value were $100 \%$ in both groups. The negative predictive value was $50 \%$ for EUS-FNB alone and 53.4 $\%$ for EUS-FNA + ROSE. In patients with an initial, non-diagnostic EUS sample, a second EUS attempt was diagnostic in $62.5 \%$ using FNB alone vs. $37.5 \%$ with EUS-FNA+ROSE $(P=0.62)$. In terms of histological yield, $88.7 \%$ of samples in the EUS-FNB alone arm were deemed histologically interpretable vs. $39.2 \%$ 
- Table 1 Comparison of demographic details between the patients managed with endoscopic ultrasound (EUS)-guided fine-needle biopsy (EUS-FNB) alone and EUS-guided fine-needle aspiration (EUS-FNA) with rapid onsite evaluation of cytopathology (ROSE).

\begin{tabular}{|c|c|c|c|c|}
\hline & Overall $(n=235)$ & EUS-FNB alone $(n=115)$ & EUS-FNA + ROSE $(n=120)$ & $P$ value \\
\hline Age, mean (SD), years & $69.7(11.8)$ & $70.2(11.0)$ & $69.2(12.7)$ & 0.52 \\
\hline Sex, female, n (\%) & $112(47.7 \%)$ & $50(43.5 \%)$ & $62(51.7 \%)$ & 0.21 \\
\hline Mean (SD) bilirubin level, $\mu \mathrm{mol} / \mathrm{L}$ & $60.2(107.1)$ & $63.6(115.9)$ & $57.4(99.0)$ & 0.68 \\
\hline Mean (SD) CA-19-9 level, IU/mL & $1693.4(5113.9)$ & $1911.4(5811.1)$ & $1489.9(4337.9)$ & 0.62 \\
\hline \multicolumn{5}{|l|}{ Tumor location, $\mathrm{n}(\%)^{1}$} \\
\hline - Uncinate/head & $136(57.9 \%)$ & $66(57.4 \%)$ & $70(58.3 \%)$ & 0.88 \\
\hline - Body/tail & $106(45.1 \%)$ & $53(46.1 \%)$ & $53(44.2 \%)$ & 0.77 \\
\hline Mean (SD) tumor size on CTscan, mm & $32.8(15.5)$ & $34.3(15.6)$ & $31.4(15.3)$ & 0.16 \\
\hline \multicolumn{5}{|c|}{ Tumor histology among patients with malignant diagnosis, $\mathrm{n}(\%)^{2}$} \\
\hline - Pancreatic adenocarcinoma & $173(79.7 \%)$ & $81(76.4 \%)$ & $92(82.9 \%)$ & 0.28 \\
\hline - Pancreatic neuroendocrine tumor & $17(7.8 \%)$ & $10(9.4 \%)$ & $7(6.3 \%)$ & 0.40 \\
\hline " Lymphoma & $3(1.4 \%)$ & $3(2.8 \%)$ & $0(0.0 \%)$ & 0.12 \\
\hline - Pseudopapillary & $3(1.4 \%)$ & $1(0.9 \%)$ & $2(1.8 \%)$ & $>0.99$ \\
\hline - Metastatic cancer & $10(4.6 \%)$ & $5(4.7 \%)$ & $5(4.5 \%)$ & $>0.99$ \\
\hline - Other & $4(1.8 \%)$ & $1(0.9 \%)$ & $3(2.7 \%)$ & 0.62 \\
\hline - Not determined & $7(3.2 \%)$ & $5(4.7 \%)$ & $2(1.8 \%)$ & 0.46 \\
\hline \multicolumn{5}{|l|}{ Cancer stage for cases of adenocarcinoma, $\mathrm{n}(\%)$} \\
\hline - Stage I & $34(19.6 \%)$ & $15(18.5 \%)$ & $19(20.7 \%)$ & 0.72 \\
\hline - Stage II & $19(11.0 \%)$ & $8(9.9 \%)$ & $11(12.0 \%)$ & 0.66 \\
\hline - Stage III & $47(27.2 \%)$ & $22(27.2 \%)$ & $25(27.2 \%)$ & 0.99 \\
\hline - Stage IV & $53(30.6 \%)$ & $31(38.3 \%)$ & $22(23.9 \%)$ & 0.04 \\
\hline - Not determined & $20(11.6 \%)$ & $5(6.2 \%)$ & $15(16.3 \%)$ & 0.18 \\
\hline \multicolumn{5}{|l|}{ Cancer stage for cases of neuroendocrine tumor, n (\%) } \\
\hline - Stagel & $2(11.8 \%)$ & $1(10.0 \%)$ & $1(14.3 \%)$ & $>0.99$ \\
\hline - Stage II & $2(11.8 \%)$ & $1(10.0 \%)$ & $1(14.3 \%)$ & $>0.99$ \\
\hline - Stage III & $3(17.7 \%)$ & $2(20.0 \%)$ & $1(14.3 \%)$ & $>0.99$ \\
\hline - Stage IV & $4(23.5 \%)$ & $2(20.0 \%)$ & $2(28.6 \%)$ & $>0.99$ \\
\hline - Not determined & $6(35.3 \%)$ & $4(40.0 \%)$ & $2(28.6 \%)$ & $>0.99$ \\
\hline Surgical resection (1 patient missing), n (\%) & $44(18.9 \%)$ & $19(16.8 \%)$ & $25(20.8 \%)$ & 0.43 \\
\hline Chemotherapy (48 patients missing), n (\%) & $110(59.1 \%)$ & $52(55.9 \%)$ & $58(62.4 .0 \%)$ & 0.37 \\
\hline Death within 6 months (20 patients missing), $\mathrm{n}(\%)$ & $58(27.0 \%)$ & $33(30.8 \%)$ & $25(22.9 \%)$ & 0.19 \\
\hline
\end{tabular}

in the EUS-FNA + ROSE arm $(P<0.001)$ ( $\triangleright$ Fig. 2). Moreover, high quality histological material was obtained in $61.7 \%$ of EUS-FNB alone vs. $12.5 \%$ of EUS-FNA + ROSE $(P<0.001)$. Standard stepwise multivariable analysis did not identify any independent predictors of diagnostic accuracy.
Overall, the cost-minimization analysis suggested that EUSFNB is slightly more costly than EUS-FNA + ROSE: $+\$ 45$ in the USA and $+\$ 102$ in Canada ( $\triangleright$ Table 4). The estimate of the marginal equipment cost of the EUS-FNB intervention is more expensive than the EUS-FNA + ROSE one $(+\$ 82$ in the USA and $+\$ 115$ in Canada). The estimate of the professional cost how- 
- Table 2 Comparison of the endoscopic ultrasound (EUS) characteristics of the tumors that were managed with EUS-guided fine-needle biopsy (EUS-FNB) alone and EUS-guided fine-needle aspiration (EUS-FNA) with rapid onsite evaluation of cytopathology (ROSE).

\begin{tabular}{|c|c|c|c|c|}
\hline & Overall $(n=235)$ & EUS-FNB alone $(n=115)$ & EUS-FNA with ROSE $(n=120)$ & $P$ value \\
\hline Size on EUS, mean (SD), mm & $29.9(12.6)$ & $30.4(13.3)$ & $29.3(12.0)$ & 0.52 \\
\hline \multicolumn{4}{|l|}{ Tumor margins ( 12 cases missing), $\mathrm{n}(\%)$} & \multirow[t]{3}{*}{0.82} \\
\hline - Well delineated & $97(43.5 \%)$ & $47(42.7 \%)$ & $50(44.2 \%)$ & \\
\hline - Poorly delineated & $126(56.5 \%)$ & $63(57.3 \%)$ & $63(55.8 \%)$ & \\
\hline \multicolumn{4}{|l|}{ Echogenicity (10 cases missing), $\mathrm{n}(\%)$} & \multirow[t]{3}{*}{0.46} \\
\hline - Heterogenous & $111(49.3 \%)$ & $53(46.9 \%)$ & $58(51.8 \%)$ & \\
\hline - Homogenous & $114(50.7 \%)$ & $60(53.1 \%)$ & $54(48.2 \%)$ & \\
\hline \multicolumn{4}{|l|}{ Size of needle (6 cases missing), $n$ (\%) } & \multirow[t]{3}{*}{$<0.01$} \\
\hline . 25 gauge & $70(30.6 \%)$ & $5(4.2 \%)$ & $65(56.0 \%)$ & \\
\hline - 22 gauge & $159(69.4 \%)$ & $108(95.6 \%)$ & $51(44.0 \%)$ & \\
\hline Mean (SD) number of needle passes & $2.6(0.9)$ & $2.3(0.6)$ & $3.0(1.1)$ & $<0.01$ \\
\hline \multicolumn{4}{|l|}{ Technique (18 cases missing), $\mathrm{n}(\%)$} & \multirow[t]{4}{*}{0.49} \\
\hline - No suction/stylet slow pull & $141(65.0 \%)$ & $75(68.8 \%)$ & $66(61.1 \%)$ & \\
\hline - Suction technique & $33(15.2 \%)$ & $15(13.8 \%)$ & $18(16.7 \%)$ & \\
\hline - Stylet slow pull & $43(19.8 \%)$ & $19(17.4 \%)$ & $24(22.2 \%)$ & \\
\hline Mean (SD) procedure time, minutes & $21.0(9.6)$ & $19.3(8.0)$ & $22.7(10.8)$ & $<0.01$ \\
\hline
\end{tabular}

- Table 3 Diagnostic characteristics for sampling of solid pancreatic lesions with endoscopic ultrasound (EUS)-guided fine-needle biopsy (EUS-FNB) alone or EUS-guided fine-needle aspiration (EUS-FNA) with rapid onsite evaluation of cytopathology (ROSE). All results are shown as percentages ( $95 \%$ confidence intervals).

\begin{tabular}{|l|l|l|l|l|}
\hline & All & EUS-FNB & EUS-FNA + with ROSE \\
\hline Accuracy & $92.8 \%(89.4 \%-96.1 \%)$ & $92.2 \%(86.6 \%-96.9 \%)$ & $93.3 \%(88.8 \%-97.9 \%)$ \\
\hline Sensitivity & $93.1 \%(88.9 \%-96.1 \%)$ & $92.5 \%(85.7 \%-96.7 \%)$ & $96.5 \%(93.0 \%-98.6 \%)$ \\
\hline Specificity & $100.0 \%(79.4 \%-100.0 \%)$ & $100.0 \%(63.1 \%-100.0 \%)$ & $100.0 \%(63.1 \%-100.0 \%)$ \\
\hline Positive predictive value & $100.0 \%$ & $100.0 \%$ & $100.0 \%$ \\
\hline Negative predictive value & $51.6 \%(39.6 \%$-63.5\%) & $50.0 \%(33.9 \%-96.9 \%)$ & $53.3 \%(35.6 \%-70.3 \%)$ \\
\hline
\end{tabular}

ever is higher for EUS FNA + ROSE than for EUS-FNB alone ( $\$ 37$ in the USA and \$14 in Canada).

\section{Discussion}

Consistent with several previous reports, our results show that EUS-FNA + ROSE can achieve diagnostic accuracy of $>90 \%$. Indeed, studies have shown that ROSE can increase the diagnostic capability of EUS by $10 \%-15 \%[3,4]$. The adoption of ROSE has however been limited outside of the USA and expert centers [8] owing to resource constraints and the emergence of conflicting RCT data regarding its benefits when compared to EUS-FNA alone $[9,18]$. Indeed, an international survey of endoscopists from 29 countries demonstrated that only $27.9 \%$ of them had access to ROSE [19]. It is important to note however that the studies that failed to show a benefit for ROSE used EUS-FNA with seven needle passes as the active comparator $[9,18]$, which is likely not representative of most clinical practice [7].

Our data suggest that FNB with the fork-tip needle can achieve excellent diagnostic accuracy of $92.2 \%$ without the need for ROSE and with a mean of only 2.3 passes. Also, EUSFNB alone was associated with a shorter procedure time than EUS-FNA with ROSE, with low rates of adverse events. Although the availability of ROSE confers certain advantages, including immediate diagnosis that can lead to more efficient downstream care, the advent of EUS-FNB obviates the need for ROSE for tissue diagnosis of solid pancreatic lesions in most settings and represents an easily implementable technology for 


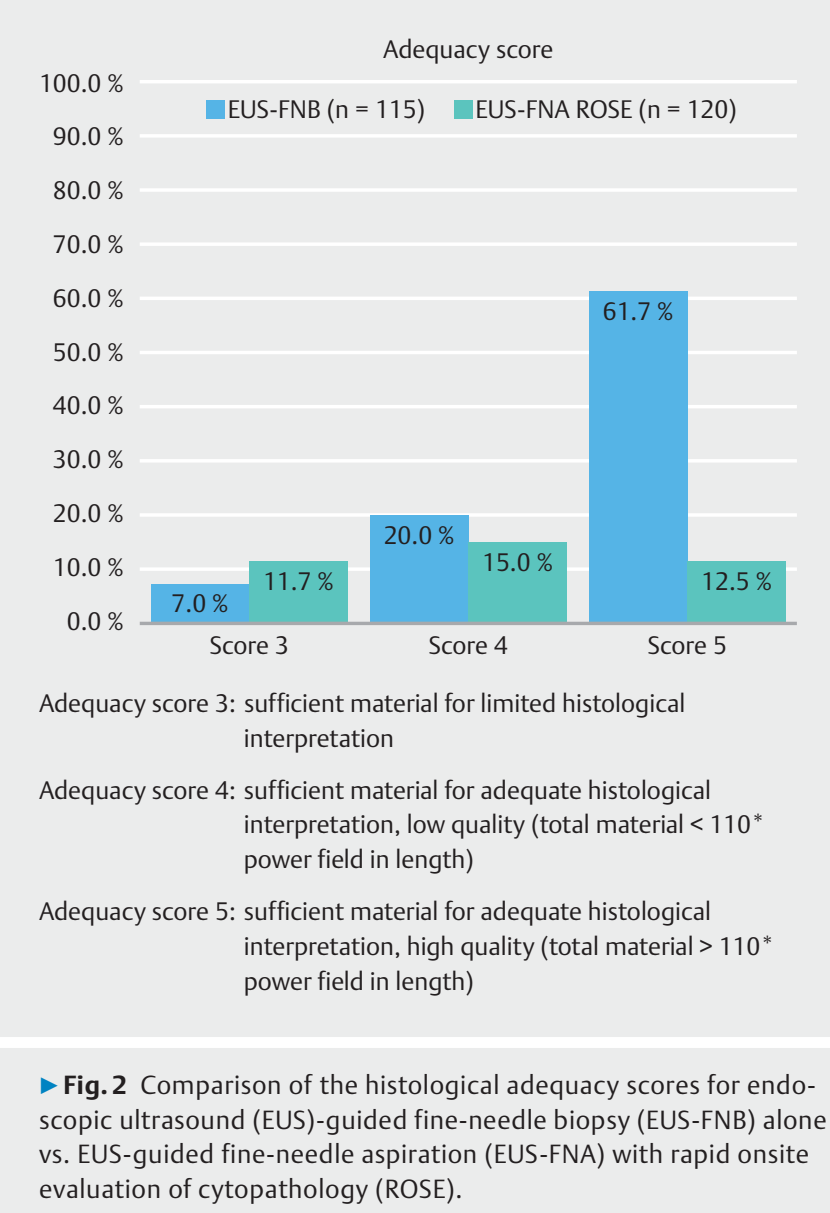

Adequacy score 3: sufficient material for limited histological interpretation

Adequacy score 4: sufficient material for adequate histological interpretation, low quality (total material $<110^{*}$ power field in length)

Adequacy score 5: sufficient material for adequate histological interpretation, high quality (total material $>110^{*}$ power field in length)

Fig. 2 Comparison of the histological adequacy scores for endoscopic ultrasound (EUS)-guided fine-needle biopsy (EUS-FNB) alone vs. EUS-guided fine-needle aspiration (EUS-FNA) with rapid onsite evaluation of cytopathology (ROSE).

institutions to employ in improving their EUS practice. To our knowledge, while there have been a number of randomized trials [20,21] and small-scale prospective studies [22] comparing EUS-FNB with EUS-FNA in different clinical indications, this is the first large-scale RCT to assess EUS-FNB alone vs. EUS-FNA with ROSE specifically for solid pancreatic lesions.

Our data also demonstrate an excellent histological yield of $87.5 \%$ with EUS-FNB. One of the most pivotal questions regarding EUS-FNB is whether or not it can acquire more DNA and tissue architecture than traditional EUS-FNA for gene sequencing and ancillary molecular studies in the growing field of precision medicine. Bang et al. [22] recently showed greater total tissue volume, higher rate of retained architecture, and better adequacy for immunohistochemistry testing with the Franseen-tip FNB needle when compared with EUS-FNA in 71 patients. Our excellent histological adequacy yield for FNB further supports the notion that the new generation of cutting FNB needles can achieve good histological specimens, with intact architecture.

Importantly, it should be remembered however that the aim of EUS-FNA is not to acquire histology, because it is processed with the goal of obtaining high quality cells not tissue architecture [23]. As such, although histology may be advantageous for immunohistochemistry testing, the question as to whether EUS-FNB can obtain better DNA for gene sequencing is unknown. In fact, some data suggest that EUS-FNA can acquire better intact DNA than EUS-FNB [23]. Therefore, although the histological yield of EUS-FNB is excellent, its role in personalized medicine remains to be determined.

In addition to its excellent diagnostic performance, the forktip FNB needle required substantially fewer needle passes, along with having a shorter procedure time, which may have important impacts on the efficiency of care in clinical practice. Although costs appear to favor EUS-FNA with ROSE, this difference (+\$45 in the USA and $+\$ 102$ in Canada) is marginal and unlikely to have a significant impact on hospital budgets in North America. More importantly, the adoption of the FNB needle is much more easily implemented in clinical practice than a ROSE program. Nevertheless, the presence of ROSE can confer several advantages and will likely maintain its important role in expert centers. For example, the ability to make an immediate preliminary diagnosis can allow for more timely subsequent care, including celiac neurolysis and biliary drainage. Early celiac neurolysis has been shown to lead to better pain control [24], while immediate tissue diagnosis optimizes stent selection if biliary drainage is performed during the same session. The availability of ROSE is however limited outside of expert centers. Therefore, EUS-FNB is likely to be the preferred modality at most institutions because it does not require the presence of ROSE to achieve excellent diagnostic accuracy, while being comparable in cost.

The limitations of this study include the lack of double blinding. As with most interventional trials, blinding of the operator is not feasible. In addition, the pathologists were also not blinded to the type of needle used. In order to limit bias, patients and study personnel involved in the data analysis were blinded to the treatment allocation. Also, the criteria used for the final diagnosis of malignant or benign disease do not rely solely on the gold standard of surgical pathological confirmation, so there is a small risk for misclassification. However, given that most pancreatic cancer patients do not undergo surgical resection, these criteria were judiciously adopted with some modification from previous publication [9] to be as accurate as possible. In addition, molecular profiling of samples would have been a better end point than histological grading to assess the adequacy of samples for personalized medicine. Currently, molecular profiling in pancreatic cancer has not however had the success of other tumors, such as lung cancer, largely because of tumor gene heterogeneity. Therefore, there is no well-established molecular panel to test and whole genome tumor sequencing is not fiscally feasible.

The strengths of this study include the randomized and multicenter design, involving both tertiary and secondary centers and endoscopists with different levels of experience, thereby strengthening our findings in terms of validity and generalizability. To our knowledge, it is also the first multicenter RCT comparing EUS-FNB alone with EUS-FNA + ROSE specifically for solid pancreatic lesions.

In conclusion, our multicenter RCT demonstrates comparable, excellent diagnostic performance between EUS-FNB without ROSE and EUS-FNA with ROSE in solid pancreatic lesions. In addition, EUS-FNB was associated with fewer needle passes, a shorter procedure time, and excellent histological yield, while 
- Table4 Cost-minimization report for sampling of solid pancreatic lesions with endoscopic ultrasound (EUS)-guided fine-needle biopsy (EUS-FNB) with a fork-tip needle alone or EUS-guided fine-needle aspiration (EUS-FNA) with rapid onsite evaluation of cytopathology (ROSE). All values have been rounded to the nearest unit.

\begin{tabular}{|c|c|c|c|c|}
\hline & \multicolumn{2}{|l|}{ USA $^{1}$} & \multicolumn{2}{|l|}{ Canada $^{2}$} \\
\hline & EUS-FNA + ROSE & EUS-FNB & EUS-FNA+ROSE & EUS-FNB \\
\hline \multicolumn{5}{|l|}{ Professional component } \\
\hline \multicolumn{5}{|l|}{ Physician fees } \\
\hline - Endoscopist fees & 210 & 210 & 305 & 305 \\
\hline - Anesthetist fees & 200 & 178 & - & - \\
\hline \multicolumn{5}{|l|}{ Nursing cost } \\
\hline - Endoscopy nursing & 14 & 12 & 15 & 12 \\
\hline - Anesthesia nursing & 70 & 64 & - & - \\
\hline Technician EUS-FNA + ROSE & 7 & - & 11 & - \\
\hline \multicolumn{5}{|l|}{ Technical component } \\
\hline - Needle & 210 & 300 & 200 & 325 \\
\hline - Microscope (single use) & 8 & - & 10 & - \\
\hline Total & 719 & 764 & 540 & 642 \\
\hline Difference EUS-FNB vs. EUS-FNA + ROSE & - & +45 & - & +102 \\
\hline $\begin{array}{l}{ }^{1} \text { In US\$ (2019). } \\
{ }^{2} \text { In CAN\$ (2019). }\end{array}$ & & & & \\
\hline
\end{tabular}

being only slightly more costly. EUS-FNB will likely become the preferred modality at most institutions, given its ease of implementation and the lack of availability of ROSE outside of expert centers. Lastly, although EUS-FNB is associated with high quality histological samples, this does not necessarily translate to better ancillary molecular studies and its role in personalized medicine in pancreatic cancer remains to be determined.

\section{Clinical trial}

Trial Registration: ClinicalTrials.gov | Registration number (trial ID): NCT03435588 | Type of study: Multicenter randomized controlled trial

\section{Competing interests}

Yen-I Chen, Avijit Chatterjee, Ian Gan, and Fergal Donnellan are consultants for Boston Scientific. Alan Barkun is a consultant for Pendopharm Inc., Boston Scientific Inc., Olympus Inc., Cook Inc., and ATGen Inc. Clarence Wong is a consultant for Boston Scientific and Medtronic Medical. The remaining authors declare that they have no conflict of interest.

\section{References}

[1] Committee ASoP, Eloubeidi MA, Decker GA et al. The role of endoscopy in the evaluation and management of patients with solid pancreatic neoplasia. Gastrointest Endosc 2016; 83: 17-28

[2] Jenssen C, Hocke M, Fusaroli P et al. EFSUMB guidelines on interventional ultrasound (INVUS), Part IV - EUS-guided interventions: General aspects and EUS-guided sampling (Short Version). Ultraschall Med 2016; 37: 157-169

[3] Klapman JB, Logrono R, Dye CE et al. Clinical impact of on-site cytopathology interpretation on endoscopic ultrasound-guided fine needle aspiration. Am J Gastroenterol 2003; 98: 1289-1294

[4] Kulesza P, Eltoum IA. Endoscopic ultrasound-guided fine-needle aspiration: sampling, pitfalls, and quality management. Clin Gastroenterol Hepatol 2007; 5: 1248-1254

[5] Ashat M, Klair JS, Rooney SL et al. Randomized controlled trial comparing the Franseen needle with a Fork-tip needle for EUS-guided fine-needle biopsy. Gastrointest Endosc 2021; 93: 140-150

[6] Bang JY, Hebert-Magee S, Navaneethan U et al. Randomized trial comparing the Franseen and Fork-tip needles for EUS-guided fineneedle biopsy sampling of solid pancreatic mass lesions. Gastrointest Endosc 2018; 87: 1432-1438

[7] Polkowski M, Jenssen C, Kaye P et al. Technical aspects of endoscopic ultrasound (EUS)-guided sampling in gastroenterology: European Society of Gastrointestinal Endoscopy (ESGE) Technical Guideline March 2017. Endoscopy 2017; 49: 989-1006

[8] Guo J, Sahai AV, Teoh A et al. An international, multi-institution survey on performing EUS-FNA and fine needle biopsy. Endosc Ultrasound 2020; 9: 319-328 
[9] Wani S, Mullady D, Early DS et al. The clinical impact of immediate onsite cytopathology evaluation during endoscopic ultrasound-guided fine needle aspiration of pancreatic masses: a prospective multicenter randomized controlled trial. Am J Gastroenterol 2015; 110: 14291439

[10] Cotton PB, Eisen GM, Aabakken L et al. A lexicon for endoscopic adverse events: report of an ASGE workshop. Gastrointest Endosc 2010; 71: $446-454$

[11] Gerke H, Rizk MK, Vanderheyden AD et al. Randomized study comparing endoscopic ultrasound-guided Trucut biopsy and fine needle aspiration with high suction. Cytopathology 2010; 21: 44-51

[12] Drummond MF, Sculpher M], Torrance GW et al. Methods for the economic evaluation of health care programmes.2nd ednOxford University Press; 2005: 379

[13] Centers for Medicare and Medicaid Services. Accessed: June 2020. Available from: https://www.cms.gov/

[14] U.S. Bureau of Labor Statistics. Accessed: June 2020. Available from: http://www.bls.gov/

[15] Schedule of Benefits and fees, Ministry of Health, Ontario. Accessed: June 2020. Available from: http: //www.health.gov.on.ca/en/pro/ programs/ohip/sob/

[16] Statistics Canada. Accessed: June 2020. Available from: https://www. statcan.gc.cal

[17] Purchasing Department, McGill University Health Center, Montreal, Canada. Accessed: June 2020. Available from: https://muhc.ca/glen
[18] Lee LS, Nieto J, Watson RR et al. Randomized noninferiority trial comparing diagnostic yield of cytopathologist-guided versus 7 passes for EUS-FNA of pancreatic masses. Dig Endosc 2016; 28: 469-475

[19] Dumonceau JM, Koessler T, van Hooft JE et al. Endoscopic ultrasonography-guided fine needle aspiration: Relatively low sensitivity in the endosonographer population. World J Gastroenterol 2012; 18: 23572363

[20] Cheng B, Zhang Y, Chen Q et al. Analysis of fine-needle biopsy vs fineneedle aspiration in diagnosis of pancreatic and abdominal masses: a prospective, multicenter, randomized controlled trial. Clin Gastroenterol Hepatol 2018; 16: 1314-1321

[21] van Riet PA, Larghi A, Attili F et al. A multicenter randomized trial comparing a 25-gauge EUS fine-needle aspiration device with a 20gauge EUS fine-needle biopsy device. Gastrointest Endosc 2019; 89: 329-339

[22] Bang JY, Hebert-Magee S, Navaneethan U et al. EUS-guided fine needle biopsy of pancreatic masses can yield true histology. Gut 2018; 67: 2081-2084

[23] Heymann JJ, Siddiqui MT. Ancillary techniques in cytologic specimens obtained from solid lesions of the pancreas: a review. Acta Cytol 2020; 64: 103-123

[24] Wyse JM, Carone M, Paquin SC et al. Randomized, double-blind, controlled trial of early endoscopic ultrasound-guided celiac plexus neurolysis to prevent pain progression in patients with newly diagnosed, painful, inoperable pancreatic cancer. J Clin Oncol 2011; 29: 35413546 